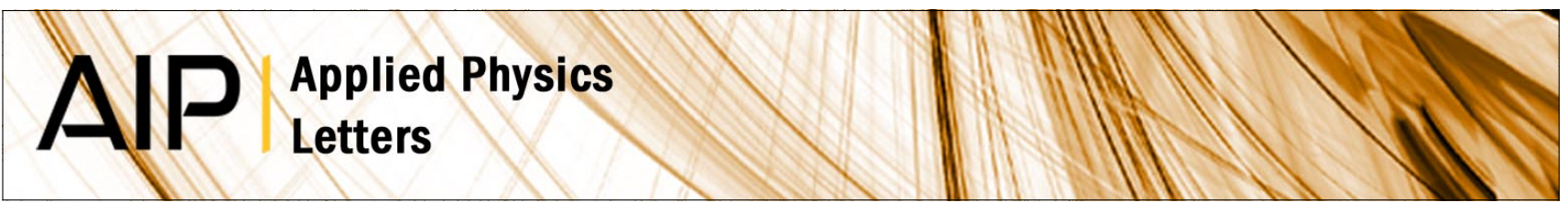

\title{
In situ analysis of free radicals from the photodecomposition of hydrogen peroxide using a frequency-mixing magnetic detector
}

Hyobong Hong, Hans-Joachim Krause, Kibong Song, and Chel-Jong Choi

Citation: Appl. Phys. Lett. 101, 054105 (2012); doi: 10.1063/1.4742740

View online: http://dx.doi.org/10.1063/1.4742740

View Table of Contents: http://apl.aip.org/resource/1/APPLAB/v101/i5

Published by the American Institute of Physics.

Additional information on Appl. Phys. Lett.

Journal Homepage: http://apl.aip.org/

Journal Information: http://apl.aip.org/about/about_the_journal

Top downloads: http://apl.aip.org/features/most_downloaded

Information for Authors: http://apl.aip.org/authors

\section{ADVERTISEMENT}

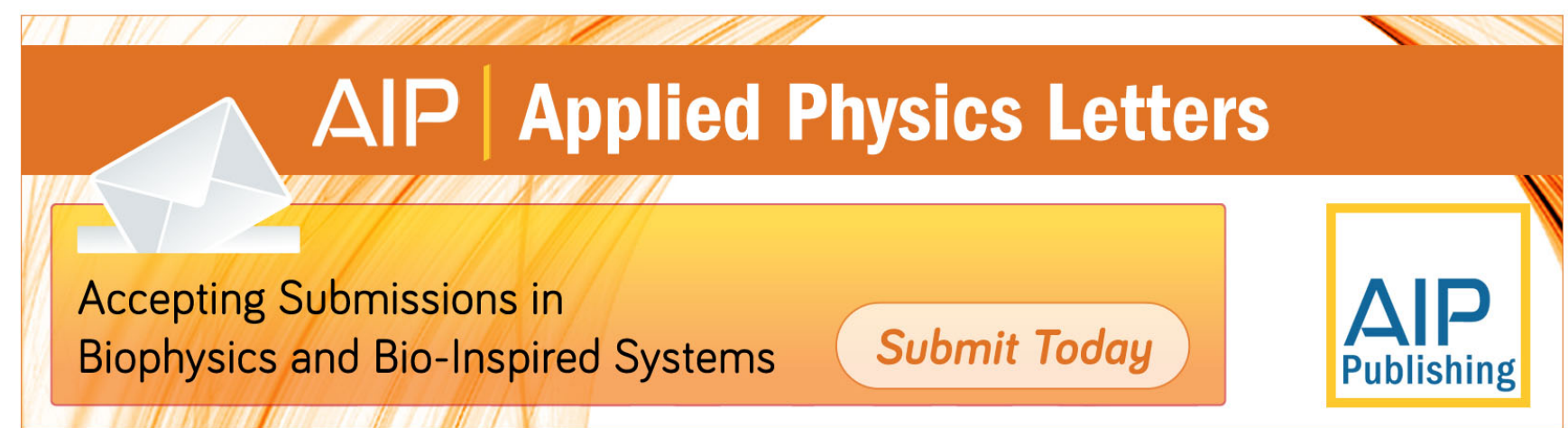




\title{
In situ analysis of free radicals from the photodecomposition of hydrogen peroxide using a frequency-mixing magnetic detector
}

\author{
Hyobong Hong, ${ }^{1}$ Hans-Joachim Krause, ${ }^{2}$ Kibong Song, ${ }^{1}$ and Chel-Jong Choi ${ }^{3,4, a)}$ \\ ${ }^{1}$ IT Convergence Service Core Research Team, Electronics and Telecommunications Research Institute, \\ Daejeon 305-700, South Korea \\ ${ }^{2}$ Peter Grünberg Institute (PGI-8), Forschungszentrum Jülich, D-52425 Jülich, Germany \\ ${ }^{3}$ School of Semiconductor and Chemical Engineering, Semiconductor Physics Research Center, \\ Chonbuk National University, Jeonju 561-756, South Korea \\ ${ }^{4}$ Department of BIN Fusion Technology, Chonbuk National University, Jeonju 561-756, South Korea
}

(Received 31 March 2012; accepted 23 July 2012; published online 3 August 2012)

\begin{abstract}
We present an analytical method for the real-time detection of free radicals from the photodecomposition (ultraviolet radiation, $\lambda=254 \mathrm{~nm}$ ) of hydrogen peroxide $\left(\mathrm{H}_{2} \mathrm{O}_{2}\right)$ using frequency-mixing magnetic detection. We monitored the free radicals in situ without catalysts or probe molecules. Both water and $\mathrm{H}_{2} \mathrm{O}_{2}$ produced frequency-mixing signals under UV radiation, but the water signal was much weaker. The root mean square amplitude of the frequency-mixing signal was found to depend on the initial $\mathrm{H}_{2} \mathrm{O}_{2}$ concentration. Considering the physical properties of the reactants, the frequency-mixing signal is attributed to the generation of paramagnetic free radicals by the photodecomposition of $\mathrm{H}_{2} \mathrm{O}_{2}$. (C) 2012 American Institute of Physics. [http://dx.doi.org/10.1063/1.4742740]
\end{abstract}

Since the discovery of free radicals by Moses Gomberg in $1900,{ }^{1}$ these species have been studied extensively because of their wide-ranging applications in various fields. Generally, free radicals have an open-shell configuration with unpaired electrons, making them paramagnetic species. ${ }^{2}$ Previously, considerable effort was made to investigate the generation, degradation, and kinetics of free radicals using analytical methods such as titration, colorimetry, fluorescence, and biochemical methods using peroxides. ${ }^{3-7}$ However, these methods suffer from the contamination of the samples caused by the probe chemicals or enzymes. For the avoidance of this problem, samples should be collected and brought to the laboratory or to the location of the analytical instrument. Furthermore, considering the instability and short lifetime of free radicals, the previous analytical methods are not capable of revealing the characteristic behavior of free radicals directly. To date, several methods enabling the detection of traces of magnetic materials have been employed to monitor free radicals without using probe molecules. These include techniques such as electron spin resonance (ESR) spectroscopy and nuclear magnetic resonance (NMR) spectroscopy. ${ }^{8-10}$ However, it is very difficult to operate and maintain these instruments under field conditions or even in a standard laboratory environment.

In this study, we propose a technique for the in situ monitoring of the free radicals produced from the photodecomposition of $\mathrm{H}_{2} \mathrm{O}_{2}$ (Sigma-Aldrich, Trace analysis Grade, no stabilizer) caused by ultraviolet (UV) radiation at $254 \mathrm{~nm}$ with an output power of $5.5 \mathrm{~W}$, as illustrated in Fig. 1. This technique involves the use of a portable detector based on two-frequency magnetic-field excitation. ${ }^{11}$ In the detector, the magnetic materials are excited by magnetic fields at two different frequencies generated in the measurement head.

\footnotetext{
${ }^{\text {a) }}$ Author to whom correspondence should be addressed. Electronic mail: cjchoi@chonbuk.ac.kr.
}

The detector measures the magnetic-field component at a sum frequency generated by the sample. Thus, the technique is sensitive to nonlinearities in the magnetization curve of the sample. The detection coil consists of a balanced arrangement of a measuring coil and a reference coil. ${ }^{11,12}$ The magnetic materials in the measurement coil are detected and quantified by acquiring the difference in magnetic field between the measuring coil and the empty reference coil. Compared to ESR spectrometry, which is the most commonly used method for free radical analysis, the frequency mixing method is much simpler. Frequency mixing appears to be particularly useful for direct assessment of short-lived free radicals such as hydroxyl and superoxide, which are usually analyzed by spin trapping techniques. The temperature of the heat exchanger was adjusted to $20^{\circ} \mathrm{C}$ in order to keep the reactor at a constant temperature.

Figure 2(a) shows the plots of frequency-mixing signal intensity as a function of measurement time for pure water and an aqueous solution of $\mathrm{H}_{2} \mathrm{O}_{2}$ (concentration of $30 \%$ ). For both samples, the frequency-mixing signals are insignificant in the absence of UV radiation. However, after the UV lamp was turned on, a considerable frequency-mixing signal was generated for both the pure water and the $30 \% \mathrm{H}_{2} \mathrm{O}_{2}$ solution, though the signal intensity measured from pure water was much weaker than that from $\mathrm{H}_{2} \mathrm{O}_{2}$. According to previous research, the frequency-mixing signal is specific to nonlinearities in the magnetization curve. ${ }^{11,12}$ These nonlinearities are particularly pronounced for superparamagnetic or ferromagnetic substances under magnetic fields close to their saturation value, but they may also appear for paramagnetic substances. It was previously reported that hydroxyl radicals are formed by the photodecomposition of $\mathrm{H}_{2} \mathrm{O}_{2}$ irradiated by light sources with wavelengths of less than $380 \mathrm{~nm}$, e.g., at $254 \mathrm{~nm} .{ }^{13,14}$ In addition, according to the Haber-Weiss mechanism, the decomposition of $\mathrm{H}_{2} \mathrm{O}_{2}$ by electromagnetic radiation such as UV illumination leads to the formation of water and oxygen 
(a)

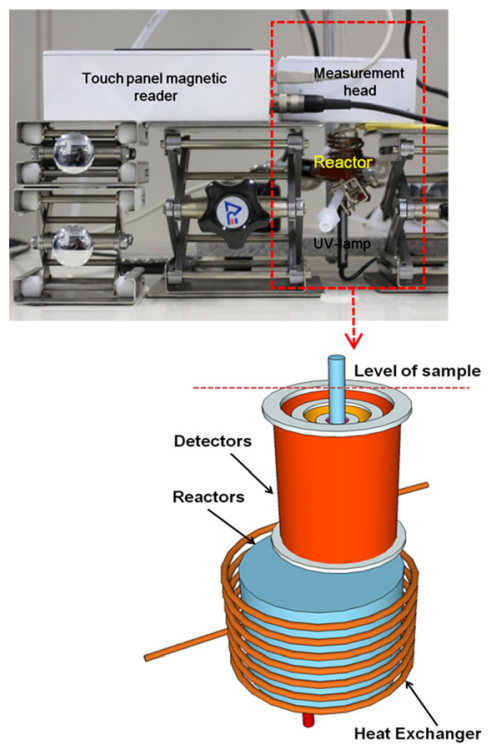

(b)

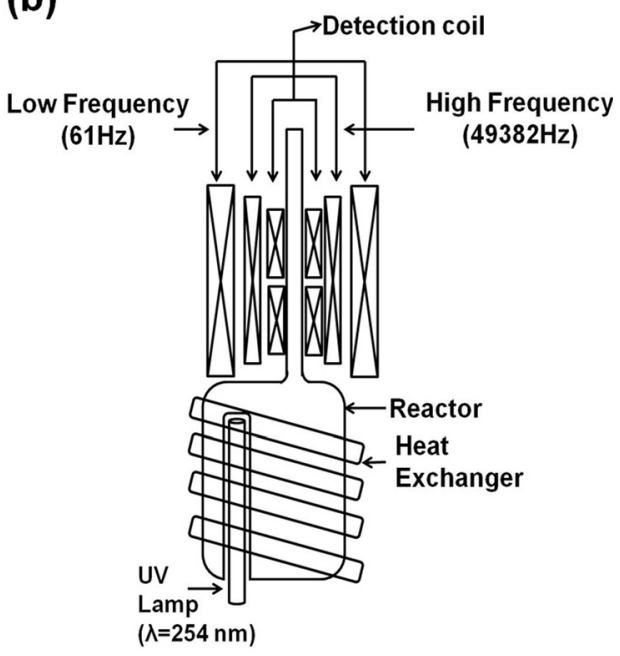

FIG. 1. (a) Experimental apparatus employed for measuring the photodecomposition of $\mathrm{H}_{2} \mathrm{O}_{2}$. Pure water or $\mathrm{H}_{2} \mathrm{O}_{2}$ solution is filled to the sample level. (b) Schematic diagram showing the generation of signals from the sample by frequency-mixing magnetic forces. The theoretical details of the experimental instrumentation are described in Ref. 11. through a propagation cycle initiated by the hydroxyl radicals formed via hemolytic cleavage of the central OH-HO bond. ${ }^{15}$ Similarly, the radical chain mechanism of the photodecomposition of $\mathrm{H}_{2} \mathrm{O}_{2}$ in pure water shows that $\mathrm{UV}$ radiation can generate $\mathrm{H}_{2} \mathrm{O}_{2}$, oxygen, and several free-radical species such as hydroxyl, hydroperoxyl, and peroxide radicals. ${ }^{16,17}$ Photodecomposition of $\mathrm{H}_{2} \mathrm{O}_{2}$ is a chain reaction with fast generation and degradation of free radicals. It is well known that water and $\mathrm{H}_{2} \mathrm{O}_{2}$ are diamagnetic substances. In contrast, the possible byproducts such as triplet oxygen molecules and free radicals are paramagnetic substances because of the unpaired electrons in their outer shell. ${ }^{18}$ Thus, the presence of oxygen molecules and free radicals produced from the photodecomposition of $\mathrm{H}_{2} \mathrm{O}_{2}$ could be the main cause of the generation of the frequency-mixing signal. Due to the differential configuration of the two serially connected pickup coils with opposite winding orientation, the sign of the signal depends on the coil in which it is picked up. Paramagnetic radicals in the liquid phase inside the bottom pickup coil yield a positive signal, whereas radicals in the gaseous phase in the top pickup coil give rise to a negative signal. We assume that the observed alternating sign of the frequency mixing signal can be attributed to the rapid change of the ratio of radicals in the upper and lower pickup coil. However, considering the melting temperature of oxygen, oxygen molecules formed through the photodecomposition of $\mathrm{H}_{2} \mathrm{O}_{2}$ are in the gas phase and are diamagnetic, so they will make a negligible contribution to the occurrence of the frequency-mixing signal. In fact, in subsequent experiments, in which pure oxygen gas (99.99\%) was passed through the reactor with and without UV radiation, no significant signals were observed, as shown in Fig. 2(b). Thus, the considerable frequency-mixing signal generated during the photodecomposition of $\mathrm{H}_{2} \mathrm{O}_{2}$ originates mainly from the free radicals formed by this reaction. In particular, the significant frequency-mixing signal measured even from pure water under UV radiation indicates that free radicals are also generated through the photodecomposition of pure water, though the number of free radicals created by the photodecomposition of pure water is much lower than that from the photodecomposition of $\mathrm{H}_{2} \mathrm{O}_{2}$ solution. ${ }^{19}$

Figure 3 shows the calibration curve obtained as a function of the concentration of $\mathrm{H}_{2} \mathrm{O}_{2}(0 \%-30 \%)$ in aqueous solution. For the determination of the calibration curve, the root mean square (RMS) amplitude of the frequency-mixing signal measured between 61 and $600 \mathrm{~s}$ (after UV irradiation in Fig. 2(a)) was subtracted from that measured between 1
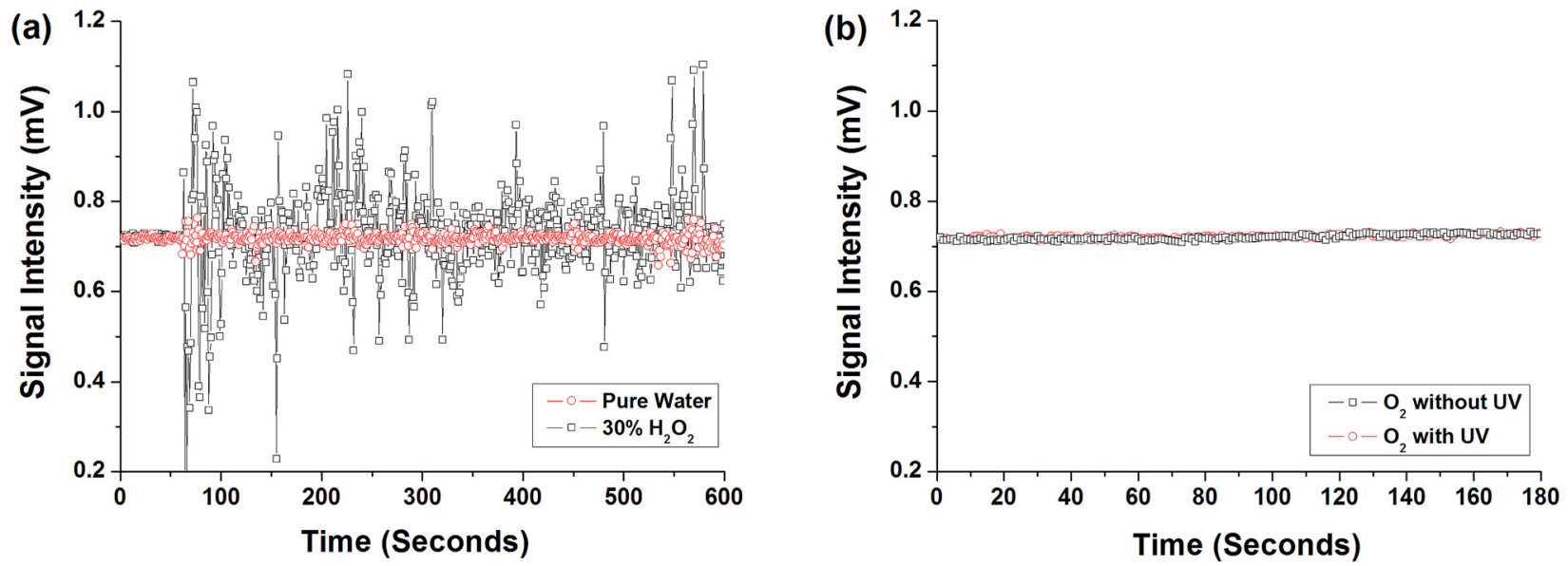

FIG. 2. (a) Comparison of the frequency-mixing signals from pure water and $30 \% \mathrm{H}_{2} \mathrm{O}_{2}$ solution. (b) Frequency-mixing signals measured from pure oxygen gas $(99.99 \%)$ with and without UV irradiation. 


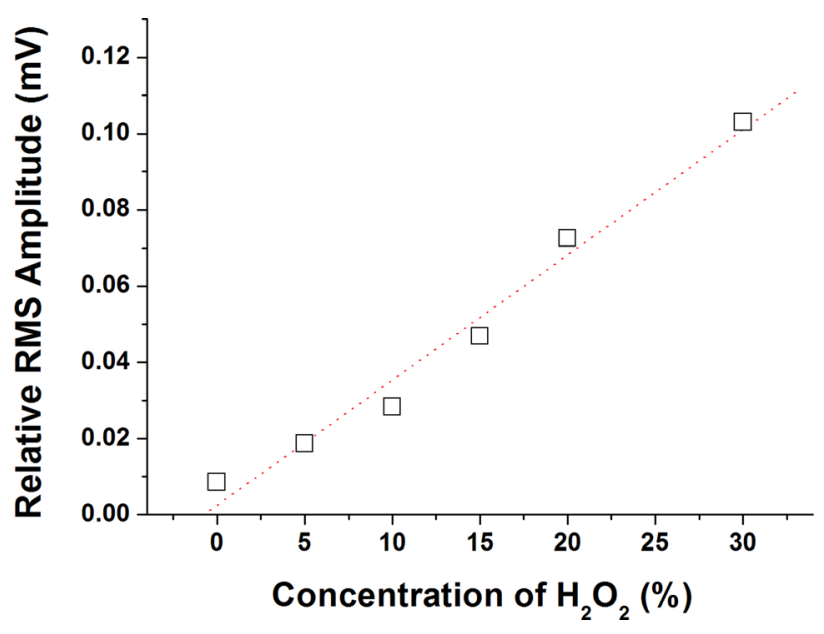

FIG. 3. Plot of relative RMS amplitude of frequency-mixing signal as a function of $\mathrm{H}_{2} \mathrm{O}_{2}$ concentration.

and $60 \mathrm{~s}$ (before UV irradiation in Fig. 2(a)). The plot clearly shows a strong linear correlation between the relative RMS amplitude of the frequency-mixing signal and the concentration of $\mathrm{H}_{2} \mathrm{O}_{2}$. This indicates that the magnetic frequencymixing technique employed in this study can be applied effectively for the simultaneous determination of the concentration of $\mathrm{H}_{2} \mathrm{O}_{2}$ and analysis of the generation of free radicals. In particular, the method used in this study enables insitu monitoring of $\mathrm{H}_{2} \mathrm{O}_{2}$ without the addition of chemicals for color development or fluorescence detection, and thus avoids contamination of the samples.

In summary, we demonstrated that frequency-mixing magnetic detection can be used to monitor the generation of free radicals from the photodecomposition of $\mathrm{H}_{2} \mathrm{O}_{2}$ in situ and determine the $\mathrm{H}_{2} \mathrm{O}_{2}$ concentration directly, without contamination of the samples by probe molecules. The frequency mixing technique needs neither a strong magnet nor a long sweeping time, thus allowing the detection of free radicals in a continuous fluidic system. The present work clearly showed that our approach is particularly useful not only for providing a better understanding of the behavior of free radicals but also for investigating the characteristics of paramagnetic substances produced by various reactions such as photochemical reactions, the Fenton reaction, or the advanced oxygen process.

This research was supported financially by the Converging Research Center Program (2011K000677) and the World Class University (WCU) Program (R31-20029) through the National Research Foundation of Korea (NRF) funded by the Ministry of Education, Science and Technology, Republic of Korea.

${ }^{1}$ M. Gomberg, Chem. Rev. 1, 91 (1924).

${ }^{2}$ T. L. Chu, G. E. Pake, D. E. Paul, J. Townsend, and S. I. Weissman, J. Phys. Chem. 57, 504 (1953).

${ }^{3}$ J. N. Rodríguez-López, D. J. Lowe, J. Hernández-Ruiz, A. N. P. Hiner, F. García-Cánovas, and R. N. F. Thorneley, J. Am. Chem. Soc. 123, 11838 (2001).

${ }^{4}$ A. Gomes, E. Fernandes, and J. L. F. C. Lima, J. Biochem. Biophys. Methods 65,45 (2005).

${ }^{5}$ J. J. Grant, B. W. Yun, and G. J. Loake, Plant J. 24, 569 (2000).

${ }^{6}$ Q. Lu, X. Dong, L. J. Li, and X. Hu, Talanta 82, 1344 (2010).

${ }^{7}$ I. Yamazaki and L. H. Piette, J. Am. Chem. Soc. 113, 7588 (1991).

${ }^{8}$ J. C. Kertesz, W. Wolf, M. B. Wolf, and B. J. Sullivan, J. Magn. Reson. 15, 427 (1974).

${ }^{9}$ V. V. Khramtsov, V. A. Reznikov, L. J. Berliner, A. K. Litkin, I. A. Grigor'ev, and T. L. Clanton, Free Radic Biol. Med. 30, 1099 (2001).

${ }^{10}$ L. Zoia and D. S. Argyropoulos, Eur. J. Mass Spectrom. 16, 175 (2010).

${ }^{11}$ H.-J. Krause, N. Wolters, Y. Zhang, A. Offenhäusser, P. Miethe, M. H. F. Meyer, M. Hartmann, and M. Keusgen, J. Magn. Magn. Mater. 311, 436 (2007).

${ }^{12}$ M. H. Meyer, M. Hartmann, H. J. Krause, G. Blankenstein, B. MuellerChorus, J. Oster, P. Miethe, and M. Keusgen, Biosens. Bioelectron. 22, 973 (2007).

${ }^{13}$ H. Ikai, K. Nakamura, M. Shirato, T. Kanno, A. Iwasawa, K. Sasaki, Y. Niwano, and M. Kohno, Antimicrob. Agents Chemother. 54, 5086 (2010).

${ }^{14}$ J. H. Baxendale and J. A. Wilson, Trans. Faraday Soc. 53, 344 (1957).

${ }^{15}$ J. P. Kehrer, Toxicology 149, 43 (2000).

${ }^{16}$ S. Lunák and P. Sedlák, J. Photochem. Photobiol., A 68, 1 (1992).

${ }^{17}$ M. L. Mariani, M. D. Labas, R. J. Brandi, A. E. Cassano, and C. S. Zalazar, Water Sci. Technol. 61, 3026 (2010).

${ }^{18}$ J. van den Handel, Physica 18, 921 (1952).

${ }^{19}$ T. Oppenlaender, Photochemical Purification of Water and Air (WileyVCH, Weinheim, 2003), pp. 1063. 